\title{
Cardiovascular risk during hormonal treatment in patients with prostate cancer
}

This article was published in the following Dove Press journal:

Cancer Management and Research

28 February 201I

Number of times this article has been viewed

\author{
Hein Van Poppel' \\ Bertrand Tombal ${ }^{2}$ \\ 'Department of Urology, University \\ Hospital, KU Leuven, Leuven, \\ Belgium; '2Department of Urology, \\ University Hospital, UC Louvain, \\ Brussels, Belgium
}

\begin{abstract}
The objective of this review is to provide information on cardiovascular risk following androgen-deprivation therapy (ADT) in prostate cancer patients and to suggest potential prevention and management strategies. Androgen deprivation therapy can cause peripheral insulin resistance, increase fat mass and low-density lipoprotein cholesterol, and induce type 2 diabetes. While recent studies have reported an association in patients with prostate cancer between ADT and increased risk of cardiovascular events, other studies have not detected the association. However, at this time, it is plausible that ADT could increase cardiovascular risk because of the adverse effect of ADT on risk factors for cardiovascular disease. It is advisable that prostate cancer patients in whom ADT is initiated be referred to their physician, who will carefully monitor them for potential metabolic effects. Therefore, physicians should be informed about these potential side effects. This especially applies to men aged $>65$ years and those with pre-existing cardiovascular comorbidities. Adopting a healthy lifestyle including a balanced diet and regular physical activity is recommended. Patients with cardiovascular disease should receive appropriate preventive therapies, including lipid-lowering, antihypertensive, glucoselowering, and antiplatelet therapy. ADT should preferably not be unnecessarily administered to prostate cancer patients with pre-existing cardiovascular disease, certainly not to those in whom the risk of prostate cancer-specific mortality is low. The physician should carefully weigh the potential benefits of ADT against the possible risks in individual patients with prostate cancer.
\end{abstract}

Keywords: androgen-deprivation therapy, cardiovascular disease, complications, prostate cancer

\section{Introduction}

The standard treatment for locally advanced and metastatic prostate cancer is androgen-deprivation therapy (ADT), which can be achieved by means of surgical castration (orchiectomy) or the use of gonadotropin-releasing hormone agonists or antagonists.

Recent epidemiological data indicate that ADT is increasingly being used in asymptomatic patients with early-stage disease, a setting in which benefits are lacking. ${ }^{1}$ Patients are increasingly exposed to long-term ADT, and may take gonadotropinreleasing hormone agonists for more than 10 years. However, ADT is accompanied by a number of adverse effects (Table 1). ${ }^{2}$ Concerns have been raised particularly about metabolic and cardiovascular side effects of ADT because of their impact on morbidity and mortality in men with prostate cancer. The purpose of this review is to provide
Correspondence: Hendrik Van Poppel University Hospital KU Leuven,

Department of Urology,

B-3000 Leuven, Belgium

Tel +32I 6346687

Fax +3216346931

Email hendrik.vanpoppel@uz.kuleuven. ac.be 
Table I Potential side effects experienced by men receiving androgen deprivation therapy for prostate cancer

Hot flushes

Fatigue

Erectile dysfunction/decreased libido

Reduced bone mass and skeletal complications

Metabolic complications

Increased fat mass/weight gain

Cardiovascular complications

Anemia

Reduced muscle strength/fatigue

Cognitive changes/depression

information on cardiovascular risk following ADT in prostate cancer patients and to suggest potential prevention and management strategies.

\section{Methods}

Data for this review were identified on Medline using the search terms "prostate neoplasms" (MeSH), "gonadotropinreleasing hormone" (MeSH), "androgen antagonists" $(\mathrm{MeSH})$, and "cardiovascular diseases" (MeSH major topic). From the references obtained, selection was made based on the clinical relevance and importance of the article. Subsequent references were identified from the reference lists of retrieved articles. The search was limited to papers published in English between 2001 and 2010.

\section{Metabolic side effects of ADT}

Several mechanisms may contribute to cardiovascular toxicity. Prospective clinical studies have demonstrated that ADT causes dramatic changes in the patient's body mass composition and induces peripheral resistance to insulin that can induce or precipitate type 2 diabetes and dyslipidemia. ${ }^{3-8}$ ADT causes a very morbid combination of muscular atrophy (decreased lean mass) and an increase in fatty deposits, a situation known as "sarcopenic obesity". 9 Sarcopenic obesity is associated with several typical features of the metabolic syndrome, such as increased subcutaneous fat, increased high-density lipoprotein cholesterol, and increased levels of adiponectin. ${ }^{10}$ According to the National Cholesterol Education Program's Adult Treatment Panel III guidelines, a man is considered to have metabolic syndrome if he meets three of the criteria shown in Table 2. ${ }^{11}$ A cross-sectional study showed that nearly $80 \%$ of prostate cancer patients on ADT for at least 1 year had a waist circumference $\geq 102 \mathrm{~cm}$ and $55 \%$ of these patients have metabolic syndrome, predisposing them to higher cardiovascular risk. Among the components
Table 2 Definition of metabolic syndrome in men

Any three or more of the following criteria:

-Waist circumference $>102 \mathrm{~cm}$

- Triglycerides $\geq 150 \mathrm{mg} / 100 \mathrm{~mL}$

- High-density lipoprotein cholesterol $<40 \mathrm{mg} / 100 \mathrm{~mL}$

- Blood pressure $\geq 130 / 85 \mathrm{mmHg}$

- Fasting glucose $\geq 110 \mathrm{mg} / 100 \mathrm{~mL}$

Note: *According to the National Cholesterol Education Program's Adult Treatment Panel III.

of metabolic syndrome, ADT-treated men had a higher prevalence of abdominal obesity and hyperglycemia. They also had elevated triglycerides compared with controls. ${ }^{12}$ Prospective studies have shown that some of the ADT-associated changes are different from the classical metabolic syndrome changes (Table 3). ADT increases subcutaneous rather than visceral fat. ${ }^{6}$ Additionally, ADT increases rather than decreases high-density lipoprotein cholesterol and does not change waist-to-hip ratio. Finally, ADT is associated with increased serum adiponectin levels and unchanged C-reactive protein levels, while the conventional metabolic syndrome is characterized by low levels of adiponectin and increased C-reactive protein. ADT-associated metabolic changes may lead to an increased risk of cardiovascular morbidity and mortality. An increase in arterial stiffness may also contribute to the association between gonadotropin-releasing hormone agonists and increased cardiovascular-related toxicity. ${ }^{8}$ Finally, it is worth mentioning that ADT may prolong the QT interval, as reported by Garnick et al after reviewing the results of three Phase III randomized trials of different modalities of ADT. Indeed a prolonged QT interval $(>450-500 \mathrm{msec})$ is associated with a higher incidence of arrhythmias and sudden death. ADT was associated with 9-21 msec increases in the corrected QT interval among a total of 476 patients. ${ }^{13}$

Table 3 Classical metabolic syndrome changes and metabolic changes in men treated with gonadotropin-releasing hormone agonists

\begin{tabular}{|c|c|c|}
\hline & $\begin{array}{l}\text { Classical } \\
\text { metabolic } \\
\text { syndrome }\end{array}$ & $\begin{array}{l}\text { Men treated with } \\
\text { gonadotropin-releasing } \\
\text { hormone agonists }\end{array}$ \\
\hline Blood pressure & Increased & No change \\
\hline Waist circumference & Increased & Increased \\
\hline Waist-to-hip ratio & Increased & No change \\
\hline Triglycerides & Increased & Increased \\
\hline $\begin{array}{l}\text { High-density lipoprotein } \\
\text { cholesterol }\end{array}$ & Decreased & Increased \\
\hline Fat accumulation & Visceral & Subcutaneous \\
\hline Adiponectin & Decreased & Increased \\
\hline
\end{tabular}




\section{Effect on cardiovascular events and mortality}

Although there is much evidence confirming a profound effect of ADT on metabolic changes, the real impact of the rate of cardiovascular events and cardiovascular morbidity is far from clear. Several large cohort studies have examined the association between ADT and increased incidence of cardiovascular disease and diabetes. In a recent populationbased observational study of 37,443 men of all ages with nonmetastatic prostate cancer, using data from the Veterans Administration, Keating et al reported an association between gonadotropin-releasing hormone agonists and a significantly increased risk of incident diabetes (adjusted hazards ratio $[\mathrm{HR}]=1.28,95 \%$ confidence interval $[\mathrm{CI}]: 1.19-1.38)$, incident coronary heart disease (adjusted HR $=1.19,95 \%$ CI: $1.10-1.28$ ), myocardial infarction (adjusted $\mathrm{HR}=1.28$, 95\% CI: 1.08-1.52), sudden death (adjusted HR $=1.35,95 \%$ CI:1.18-1.54), and stroke (adjusted HR $=1.22,95 \%$ CI: 1.10 1.36). Combined androgen blockade is significantly associated with an increased risk of incident coronary heart disease (adjusted HR $=1.27,95 \%$ CI: $1.05-1.53$ ) and orchiectomy is associated with coronary heart disease (adjusted HR $=1.40$, 95\% CI: 1.04-1.87) and myocardial infarction (adjusted $\mathrm{HR}=2.11,95 \% \mathrm{CI}: 1.27-3.50$ ). The same study concluded that monotherapy with nonsteroidal antiandrogen was not associated with any of the toxic effects examined, therefore suggesting a potentially wider place in patients with cardiovascular toxicity. Incident coronary heart disease was defined as coronary heart disease identified at least 6 months after diagnosis in men without prevalent disease. ${ }^{14}$ These findings have been confirmed in another study from the same group using the Surveillance, Epidemiology and End Results (SEER) Medicare data $(n=73,196)$, but analyses were limited to older men ( $\geq 66$ years), and information was lacking about antiandrogens and whether ADT was associated with stroke. Gonadotropin-releasing hormone agonist use was associated with increased risk of incident diabetes (adjusted HR $=1.44, P<0.001$ ), coronary heart disease (adjusted $\mathrm{HR}=1.16, P<0.001$ ), myocardial infarction (adjusted HR $=1.11, P=0.03$ ), and sudden cardiac death (adjusted $\mathrm{HR}=1.16, P=0.004) .{ }^{15}$ Saigal et al examined the risk of cardiovascular morbidity in men $\geq 65$ years with newly diagnosed prostate cancer on ADT also using the SEER Medicare data $(n=22,816)$. They found that men who received ADT had a $20 \%$ higher risk of cardiovascular morbidity compared with a matched male population that did not receive ADT (adjusted HR $=1.20,95 \%$ CI: $1.15-1.26 ; P<0.05$ ). This increased risk was observed within the initial 12 months of ADT. ${ }^{16}$ A recent preliminary report from a populationbased, matched-cohort study found an association between continuous use of ADT for at least 6 months in older men ( $\geq 66$ years) and increased incidence of diabetes (adjusted HR $=1.16,95 \%$ CI: 1.11-1.21), but not of myocardial infarction or sudden cardiac death. ${ }^{17}$ Three retrospective cohort studies have reported a significantly increased risk of cardiovascular mortality from ADT (adjusted HR 1.16, ${ }^{15}$ $1.35,{ }^{14}$ and $\left.2.6^{18}\right)$. The studies by Keating et al have been described previously. ${ }^{14,15}$ The study by Tsai et al reported analyses of 4892 men included in the CAPSURE (Cancer of the Prostatic Urologic Research Endeavor) database. The authors suggested the association of ADT with an increased risk of cardiovascular mortality in a subset of men undergoing radical prostatectomy for localized prostate cancer (adjusted HR $=2.6,95 \%$ CI: $1.4-4.7 ; P=0.002$ ) but not in a subset of men treated with radiation therapy. ${ }^{18}$ The risk of severe cardiovascular morbidity after ADT is not equal across the population. Patients aged above 65 years have a higher risk than younger men. ${ }^{18}$ The 5 -year cumulative incidence of cardiovascular mortality was $5.5 \%$ for patients aged $\geq 65$ years who received ADT and $2 \%$ for non-ADT controls. For younger patients, the 5 -year cumulative incidence of cardiovascular mortality was $3.6 \%$ for those who received $\mathrm{ADT}$ and $2 \%$ in those not treated with $\mathrm{ADT} .{ }^{18}$ Nanda et al reported the results of a retrospective study including 5077 men with localized or locally advanced prostate cancer who were treated with or without a median of 4 months of neoadjuvant hormone therapy (gonadotropin-releasing hormone agonist and antiandrogen) followed by radiation therapy. They found that the use of neoadjuvant hormone therapy is significantly associated with an increased risk of all-cause mortality among men with a history of coronary artery disease-induced congestive heart failure or myocardial infarction, but not among men with no comorbidity or a single risk factor for coronary artery disease. In the subgroup of patients with coronary artery disease-induced congestive heart failure or myocardial infarction, 25/95 deaths were reported in ADT-treated patients $(26.3 \%)$ and 18/161 deaths in non-ADT-treated controls (11.2\%, adjusted $\mathrm{HR}=1.96,95 \% \mathrm{CI}: 1.04-3.71, P=0.04) .{ }^{19}$ D'Amico et al analyzed post hoc pooled data on 1372 patients from three randomized trials of radiation therapy with or without ADT for localized prostate cancer. They found a shorter time to fatal myocardial infarction in men aged $\geq 65$ years who received 6 months of ADT compared with men in this age group with no ADT use $(P=0.017)$. Additional evidence to support this result is needed. ${ }^{20}$ 
Interestingly, the results of these large epidemiological surveys are not supported by secondary analysis of cardiovascular events and mortality observed in prospective randomized trials evaluating the benefit of early hormone therapy, especially in combination with external beam radiation therapy. Secondary analyses of four randomized controlled studies from the Radiation Therapy Oncology Group or European Organization for Research and Treatment of Cancer (EORTC) have found no association between neoadjuvant or adjuvant ADT and cardiovascular-related mortality (Table 4). ${ }^{21-24}$ It has to be noted that none of these studies were primarily designed to assess cardiovascular mortality. A recently completed EORTC randomized study (Protocol 22961) comparing radiation therapy plus a total of 6 months ADT with radiation therapy plus a total of 3 years ADT in patients with locally advanced prostate cancer showed no significant difference in the incidence of fatal cardiac events at 5 -year follow-up ( $4.0 \%$ versus $3.0 \%$, respectively). ${ }^{25}$

Consequently, the physicians are left with that controversy opposing the "epidemiologists" and the aficionados of randomized controlled trials. But, is it important to address that controversy? It is clear enough that ADT induces anthropomorphic and metabolic changes that have been known for years to be associated with an increased cardiovascular risk. Why would it be different in prostate cancer patients receiving ADT? The more important conclusion is that physicians who prescribe ADT should be aware of these metabolic side effects, and monitor and treat them according to standard recommendations, as any primary care physician would do for any man concerned about his health. And even more important, it should remind the physician that ADT is not an innocuous treatment, and that it should be prescribed only in men needing ADT. The US Food and Drug Administration is examining the safety of gonadotropin-releasing hormone agonists for treatment of prostate cancer, and believes it is important to inform patients and health care professionals about these side effects.

In a recently published population-based study by Hemelrijck et al, 30,642 patients with prostate cancer received primary hormonal treatment, 26,432 received curative treatment, and 19,527 had surveillance. Increased relative risks of nonfatal and fatal cardiovascular disease were found among all men with prostate cancer, especially those treated with hormonal treatment. They concluded that cardiovascular disease risk should be considered when prescribing hormonal treatment, but should not be a contraindication when the expected benefit is real. ${ }^{26}$ This supports the recent science advisory from the American Heart Association, the American Cancer Society, and the American Urological Association. $^{27}$

\section{Follow-up and management of patients on ADT}

The side effects of ADT should be explained to patients and communicated to the patient's other physicians, including the primary care doctor. Physicians should be particularly attentive to identify men with cardiovascular comorbidity and those already with significant risk factors.

Prior to initiation of ADT, it is advisable that patients are screened by their primary care physician for pre-existing presence of metabolic syndrome and risk factors for cardiovascular toxicity. ${ }^{27}$ This especially applies to older men ( $>65$ years) who have a higher prevalence of comorbidities than younger men. Good medical care dictates that

Table 4 Studies with rates of cardiovascular mortality not significantly different between treatment groups

\begin{tabular}{|c|c|c|c|}
\hline Study & Study population & Treatment groups & $\begin{array}{l}\text { Number of cardiovascular } \\
\text { deaths ( } P \text { value) }\end{array}$ \\
\hline EORTC $\left.3089\right|^{21}$ & $\begin{array}{l}\text { Locally advanced or node- } \\
\text { positive prostate cancer not } \\
\text { suitable for local treatment }\end{array}$ & $\begin{array}{l}\text { Immediate ADT } \\
\text { vs deferred ADT }\end{array}$ & $\begin{array}{l}185 \\
(17.9 \%-19.7 \%)\end{array}$ \\
\hline RTOG $8610^{22}$ & Locally advanced prostate cancer & $\begin{array}{l}\text { RT + ADT (4 months) } \\
\text { vs RT alone }\end{array}$ & $\begin{array}{l}57 \\
(14 \%-11 \%, P=0.32)\end{array}$ \\
\hline RTOG $9202^{23}$ & Locally advanced prostate cancer & $\begin{array}{l}\mathrm{RT}+\mathrm{ADT} \text { ( } 28 \text { months }) \\
\text { vs RT + ADT ( } 4 \text { months) }\end{array}$ & $\begin{array}{l}185 \\
(5.9 \%-4.8 \%, P=0.58)\end{array}$ \\
\hline RTOG $\left.853\right|^{24}$ & $\begin{array}{l}\text { Locally advanced or node- } \\
\text { positive prostate cancer } \\
\text { (unfavorable prognosis) }\end{array}$ & $\begin{array}{l}R T+\text { indefinite } A D T \\
\text { vs } R T+\text { salvage } A D T \text { for } \\
\text { recurrence }\end{array}$ & $\begin{array}{l}117 \\
(8.4 \%-11.4 \%, P=0.17)\end{array}$ \\
\hline EORTC $2296 \mathrm{I}^{25}$ & Locally advanced prostate cancer & $\begin{array}{l}\text { RT + ADT (6 months) } \\
\text { vs RT + ADT ( } 3 \text { years) }\end{array}$ & $\begin{array}{l}31-25 \\
(4.0 \%-3.0 \%)\end{array}$ \\
\hline
\end{tabular}

Abbreviations: ADT, androgen-deprivation therapy; RT, radiation therapy. 
prior to initiation of ADT, physicians collect abdominal perimeter and blood pressure, and measure fasting glucose, low-density and high-density lipoprotein cholesterol, triglycerides, and ultrasensitive C-reactive protein. These tests are best repeated every 3 months during the first year of ADT because the treatment-related metabolic and body composition changes tend to arise within months of starting ADT. ${ }^{7,15,28}$ Body composition can be monitored simply using a bioimpedance balance. There are currently no optimal strategies to prevent or manage ADT-associated metabolic effects, other than existing recommendations to reduce the risk of diabetes and cardiovascular disease in the general population. Patients are encouraged to adopt a healthy lifestyle, such as a low-fat diet, regular physical exercise, smoking cessation, and moderate alcohol intake. ${ }^{28}$ Pharmacological options are considered if lifestyle modifications prove unsuccessful. Conditions like diabetes, hyperlipidemia, and hypertension should be managed. Patients with cardiovascular disease should receive appropriate preventive therapies if necessary, including lipid-lowering, antihypertensive, glucose-lowering, and antiplatelet therapies. ${ }^{27}$ The preventive role of statins, other cardioprotective agents (eg, aspirin, beta-blockers), and insulin sensitizers should be studied in men receiving ADT for prostate cancer. Toremifene, a second-generation selective receptor modulator, has shown promise for the management of ADT-associated lipid changes, but its effect on cardiovascular outcomes has not yet been evaluated.

Recent studies have highlighted the growing role of supervised resistance and aerobic exercise programs to counteract the metabolic impact of sarcopenic obesity and peripheral insulin resistance. Segal et al demonstrated that a 12-week resistance exercise intervention (three times/ week) reduced fatigue ( $P=0.002)$, improved health-related quality of life $(P=0.001)$, and increased levels of upper body $(P=0.009)$ and lower body $(P<0.001)$ muscular fitness in men with prostate cancer receiving ADT. The intervention group included 82 men, while the control group included 73 men on a waiting list to undergo the same course of physical therapy. ${ }^{29}$ These findings were supported by a study conducted by Galvao et al who examined the effects of longer progressive resistance training (20 weeks) at a rehabilitation clinic in 10 men on ADT for localized prostate cancer. Beneficial effects were increased muscle strength (upper and lower body) and endurance $(P<0.001) .{ }^{30}$ In both of the aforementioned studies, resistance training increased muscle thickness and preserved whole body lean mass, with no change in fat mass. Levels of prostate serum antigen and testosterone remained unchanged, indicating that resistance training can be safely tolerated in men receiving $\mathrm{ADT}^{30,31}$ Segal et al recently demonstrated in a randomized controlled study that resistance and aerobic exercise alleviate fatigue in men with prostate cancer receiving radiation therapy with or without ADT. Resistance exercise generated longer-term improvements and additional benefits for quality of life, strength, triglycerides, and body fat..$^{32}$ Although there are no results as yet from large randomized controlled studies of ADT-treated patients with prostate cancer assessing the impact of exercise on cardiovascular and metabolic complications, physical training in other cancer patients, as well as in healthy adults, has been found to reduce cardiovascular and metabolic diseases significantly. Two large studies in this context are under way.

One might be tempted to believe that the cardiovascular side effects of ADT could be reduced with intermittent ADT (intermittent androgen deprivation) use when compared with continuous administration of gonadotropin-releasing hormone agonists. Several trials indicated that short-term side effects, such as sexual function and quality of life, are improved during the off-treatment period, when the administration of the luteinizing hormone releasing hormone agonist is suspended. ${ }^{33}$ Whether intermittent androgen deprivation also alleviates long-term side effects is far more controversial. None of the prospective randomized trials evaluating ADT has indeed investigated the side effects of ADT. In addition, it is noteworthy that, according to d'Amico et al, cardiovascular toxicity raised already after 6 months of ADT, and that not all patients will recover a normal testosterone when interrupting the administration of the luteinizing hormone releasing hormone agonist. ${ }^{34,35}$ This is important because, in contrast with the anticancer effect that requires profound suppression of testosterone, an increased cardiovascular risk is already observed in cases of mild hypogonadism. ${ }^{36}$

In conclusion, there are still insufficient data to determine whether intermittent ADT has the potential to prevent or reverse the long-term complications associated with ADT, and this strategy should not be offered as an alternative in men requiring ADT and at high risk of cardiovascular disease. In nonmetastatic prostate cancer patients, nonsteroidal antiandrogen monotherapy offers an important advantage compared with luteinizing hormone releasing hormone agonists. It indeed increases circulating testosterone, and therefore causes fewer changes in body weight, body mass index, and percentage of fat mass and lean mass. ${ }^{37}$ 


\section{Conclusion}

At this time, there is a growing body of evidence to suggest that ADT induces change in body composition and dyslipidemia. It is plausible that ADT could increase cardiovascular risk because of the adverse effect of ADT on risk factors for cardiovascular disease. It is advisable that before initiation and during ADT treatment, men with prostate cancer are periodically checked for potential metabolic effects by their physician. A healthy lifestyle, including a balanced diet and regular physical activity, should be encouraged. Patients with known cardiovascular disease should receive appropriate preventive treatment if necessary, including lipidlowering, antihypertensive, glucose-lowering, and antiplatelet therapies. ADT should not be unnecessarily administered to prostate cancer patients with pre-existing cardiovascular disease, and certainly not to those in whom the risk of prostate cancer-specific mortality is low. In the decision about whether to initiate ADT or not, the physician treating the patient for prostate cancer should carefully weigh the potential benefits of ADT against the possible risks in the individual patient. There is a need for large prospective studies to investigate the metabolic and cardiovascular effects of ADT, and to identify populations of men at highest risk of developing these side effects, such as diabetics. The benefit/risk ratio of ADT should also be assessed in younger patients and in those with earlier-stage prostate cancer and a favorable prognosis. Further research should focus on the development of strategies to prevent or reverse the cardiovascular and metabolic toxicities associated with ADT.

\section{Disclosure}

The authors report no conflicts of interest in this work.

\section{References}

1. Heidenreich A, Aus G, Bolla M, et al. EAU guidelines on prostate cancer. Eur Urol. 2008;53:68-80.

2. Alibhai SM, Gogov S, Allibhai Z. Long-term side effects of ADT in men with non-metastatic prostate cancer: A systematic literature review. Crit Rev Oncol Hematol. 2006;60:201-215.

3. Smith JC, Bennett S, Evans LM, et al. The effects of induced hypogonadism on arterial stiffness, body composition, and metabolic parameters in males with prostate cancer. $J$ Clin Endocrinol Metab. 2001;86:4261-4267.

4. Smith MR, Finkelstein JS, McGovern FJ, et al. Changes in body composition during ADT for prostate cancer. J Clin Endocrinol Metab. 2002;87:599-603.

5. Berruti A, Dogliotti L, Terrone C, et al. Changes in bone mineral density, lean body mass and fat content as measured by dual energy $\mathrm{x}$-ray absorptiometry in patients with prostate cancer without apparent bone metastases given ADT. $J$ Urol. 2002;167:2361-2367.

6. Smith MR. Changes in fat and lean body mass during androgendeprivation therapy for prostate cancer. Urology. 2004;63:742-745.
7. Smith MR, Lee H, Nathan DM. Insulin sensitivity during combined androgen blockade for prostate cancer. J Clin Endocrinol Metab. 2006; 91:1305-1308.

8. Dockery F, Bulpitt CJ, Agarwal S, Donaldson M, Rajkumar C. Testosterone suppression in men with prostate cancer leads to an increase in arterial stiffness and hyperinsulinaemia. Clin Sci (Lond). 2003;104:195-201.

9. Galvao DA, Spry NA, Taaffe DR, et al. Changes in muscle, fat and bone mass after 36 weeks of maximal androgen blockade for prostate cancer. BJU Int. 2008;102:44-47.

10. Smith MR, Lee H, McGovern F, et al. Metabolic changes during gonadotropin-releasing hormone agonist therapy for prostate cancer: Differences from the classic metabolic syndrome. Cancer. 2008;112: 2188-2194.

11. Executive Summary of The Third Report of the National Cholesterol Education Program (NCEP) Expert Panel on Detection, Evaluation, and Treatment of High Blood Cholesterol in Adults (Adult Treatment Panel III). Expert Panel on Detection, Evaluation, and Treatment of High Blood Cholesterol in Adults. JAMA. 2001;285:2486-2497.

12. Braga-Basaria M, Dobs AS, Muller DC, et al. Metabolic syndrome in men with prostate cancer undergoing long-term androgen-deprivation therapy. J Clin Oncol. 2006;24:3979-3983.

13. Garnick MB, Pratt CM, Campion M, Shipley J. The effect of hormonal therapy for prostate cancer on the electrocardiographic QT interval: Phase 3 results following treatment with leuprolide and goserelin, alone or with bicalutamide, and the gonadotropin-releasing hormone antagonist abarelix. J Clin Oncol. 2004;22:Abstr 4578.

14. Keating NL, O'Malley AJ, Freedland SJ, Smith MR. Diabetes and cardiovascular disease during ADT: Observational study of veterans with prostate cancer. J Natl Cancer Inst. 2009;102:39-46.

15. Keating NL, O'Malley AJ, Smith MR. Diabetes and cardiovascular disease during ADT for prostate cancer. J Clin Oncol. 2006;24: 4448-4456.

16. Saigal CS, Gore JL, Krupski TL, Hanley J, Schonlau M, Litwin MS. Androgen deprivation therapy increases cardiovascular morbidity in men with prostate cancer. Cancer. 2007;110:1493-1500.

17. Alibhai SM, Duong-Hua M, Sutradhar R, et al. Impact of ADT on cardiovascular disease and diabetes. J Clin Oncol. 2009;27: $3452-3458$.

18. Tsai HK, D'Amico AV, Sadetsky N, Chen MH, Carroll PR. Androgen deprivation therapy for localized prostate cancer and the risk of cardiovascular mortality. J Natl Cancer Inst. 2007;99:1516-1524.

19. Nanda A, Chen MH, Braccioforte MH, Moran BJ, D'Amico AV. Hormonal therapy use for prostate cancer and mortality in men with coronary artery disease-induced congestive heart failure or myocardial infarction. JAMA. 2009;302:866-873

20. D'Amico AV, Denham JW, Crook J, et al. Influence of androgen suppression therapy for prostate cancer on the frequency and timing of fatal myocardial infarctions. J Clin Oncol. 2007;25:2420-2425.

21. Studer UE, Whelan P, Albrecht W, et al. Immediate or deferred androgen deprivation for patients with prostate cancer not suitable for local treatment with curative intent: European Organisation for Research and Treatment of Cancer (EORTC) Trial 30891. J Clin Oncol. 2006; 24:1868-1876.

22. Roach M 3rd, Bae K, Speight J, et al. Short-term neoadjuvant ADT and external-beam radiotherapy for locally advanced prostate cancer: Long-term results of RTOG 8610. J Clin Oncol. 2008;26:585-591.

23. Efstathiou JA, Bae K, Shipley WU, et al. Cardiovascular mortality and duration of androgen deprivation for locally advanced prostate cancer: Analysis of RTOG 92-02. Eur Urol. 2008;54:816-823.

24. Efstathiou JA, Bae K, Shipley WU, et al. Cardiovascular mortality after ADT for locally advanced prostate cancer: RTOG 85-31. J Clin Oncol. 2009;27:92-99.

25. Bolla M, de Reijke TM, van Tienhoven G, et al. Duration of androgen suppression in the treatment of prostate cancer. N Engl J Med. 2009; 360:2516-2527. 
26. Van Hemelrijck M, Garmo H, Holmberg L, et al. Absolute and relative risk of cardiovascular disease in men with prostate cancer: Results from the population-based PCBaSe Sweden. J Clin Oncol. 2010;28: 3448-3456.

27. Levine GN, D'Amico AV, Berger P, et al. Androgen-deprivation therapy in prostate cancer and cardiovascular risk: A science advisory from the American Heart Association, American Cancer Society, and American Urological Association: Endorsed by the American Society for Radiation Oncology. CA Cancer J Clin. 2010;60:194-201.

28. Shahani S, Braga-Basaria M, Basaria S. Androgen deprivation therapy in prostate cancer and metabolic risk for atherosclerosis. J Clin Endocrinol Metab. 2008;93:2042-2049.

29. Segal RJ, Reid RD, Courneya KS, et al. Resistance exercise in men receiving ADT for prostate cancer. J Clin Oncol. 2003;21:1653-1659.

30. Galvao DA, Nosaka K, Taaffe DR, et al. Resistance training and reduction of treatment side effects in prostate cancer patients. Med Sci Sports Exerc. 2006;38:2045-2052.

31. Galvao DA, Nosaka K, Taaffe DR, et al. Endocrine and immune responses to resistance training in prostate cancer patients. Prostate Cancer Prostatic Dis. 2008;11:160-165.

32. Segal RJ, Reid RD, Courneya KS, et al. Randomized controlled trial of resistance or aerobic exercise in men receiving radiation therapy for prostate cancer. J Clin Oncol. 2009;27:344-351.
33. Abrahamsson PA. Potential benefits of intermittent androgen suppression therapy in the treatment of prostate cancer: A systematic review of the literature. Eur Urol. 2010;57:49-59.

34. D'Amico AV, Renshaw AA, Loffredo B, Chen MH. Duration of testosterone suppression and the risk of death from prostate cancer in men treated using radiation and 6 months of hormone therapy. Cancer. 2007;110:1723-1728.

35. Kaku H, Saika T, Tsushima T, et al. Time course of serum testosterone and luteinizing hormone levels after cessation of long-term luteinizing hormone-releasing hormone agonist treatment in patients with prostate cancer. Prostate. 2006;66:439-444.

36. Khaw KT, Dowsett M, Folkerd E, et al. Endogenous testosterone and mortality due to all causes, cardiovascular disease, and cancer in men: European prospective investigation into cancer in Norfolk (EPIC-Norfolk) Prospective Population Study. Circulation. 2007;116: 2694-2701.

37. Smith MR, Goode M, Zietman AL, McGovern FJ, Lee H, Finkelstein JS. Bicalutamide monotherapy versus leuprolide monotherapy for prostate cancer: Effects on bone mineral density and body composition. J Clin Oncol. 2004;22:2546-2553.
Cancer Management and Research

\section{Publish your work in this journal}

Cancer Management and Research is an international, peer-reviewed open access journal focusing on cancer research and the optimal use of preventative and integrated treatment interventions to achieve improved outcomes, enhanced survival and quality of life for the cancer patient The journal welcomes original research, clinical \& epidemiological

\section{Dovepress}

studies, reviews \& evaluations, guidelines, expert opinion \& commentary, case reports \& extended reports. The manuscript management system is completely online and includes a very quick and fair peerreview system, which is all easy to use. Visit http://www.dovepress.com/ testimonials.php to read real quotes from published authors. 